\title{
Nickelates Have Their Own Superconducting Style
}

\section{Nickel oxides appear to have a fundamentally different superconductivity from that of copper oxides, according to new calculations.}

\author{
By Matteo Rini
}

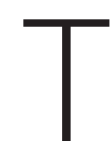

ransition-metal oxides have been intensively studied since the 1986 discovery of high-temperature (high- $T_{\mathcal{C}}$ ) superconductivity in copper oxides, or cuprates. Until 2019, high- $T_{C}$ superconductivity had only been seen in copper-based oxides. But last year, researchers discovered superconductivity in a thin nickel oxide (nickelate) film (see Trend: Entering the Nickel Age of Superconductivity). It remained unclear, however, whether the superconductivity of nickelates and cuprates was governed by the same mechanisms. New calculations by Yang Zhang at the University of Tennessee and colleagues point to important differences between the superconductivity of these two materials [1]. The findings may help in designing other nickelate superconductors and in using the material as a platform to study unconventional superconductivity.

Using density-functional theory, the team computed the electronic, magnetic, and structural properties for films of nickelates grown on a strontium titanate substrate-as in the 2019 discovery. They also performed the same calculations for

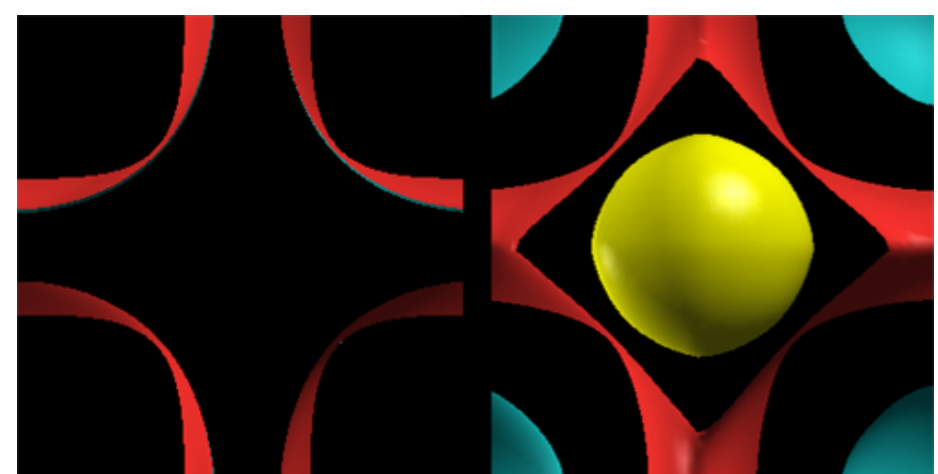

Credit: Y. Zhang et al. [1] an equivalent cuprate film. The team found a number of differences between the two materials, including opposite types of lattice distortions at the interfaces of the film. But the most striking difference relates to the electronic orbitals that likely contribute to superconductivity.

The team found that nickelates have two "active" orbitals, in which unpaired electrons can become delocalized over the film. In cuprates, only one of these orbitals is active. The team also found that the 2D gas of electrons that forms at the interface between the oxide and the substrate extends over several atomic layers for nickelates. For the equivalent cuprate structure, this gas is confined to a single layer. Together, these results call for new models for nickelate superconductivity.

Matteo Rini is the Deputy Editor of Physics.

\section{REFERENCES}

1. Y. Zhang et al., "Similarities and differences between nickelate and cuprate films grown on a $\mathrm{SrTiO}_{3}$ substrate," Phys. Rev. B 102,195117 (2020). 